\title{
Kinetics of binding, uptake and degradation of live fluorescent (DsRed) bacteria by Dictyostelium discoideum
}

\author{
Andrew Maselli, Gary Laevsky and David A. Knecht \\ Author for correspondence: David Knecht. Tel: +1 860486 2200. Fax: +1 8604864331. \\ e-mail: knecht@uconn.edu
}

Department of Molecular and Cell Biology, University of Connecticut, Storrs, CT 06269, USA
The kinetics of binding, uptake and degradation of bacteria by vegetative Dictyostelium amoeba using Escherichia coli expressing the recombinant fluorescent protein DsRed have been characterized. There are significant advantages to using DsRed-expressing bacteria for phagocytosis assays. Stable expression of the fluorescent protein, DsRed, provides living bacteria with a bright internal fluorescent signal that is degradable in the phagolysosomal pathway. Unlike assays with chemically labelled bacteria or latex beads, the bacteria are alive and possess a natural, unaltered external surface for receptor interaction. Dictyostelium cells rapidly bind and phagocytose DsRed bacteria. Pulse-chase experiments show that the signal derived from DsRed is degraded with a half-life of approximately $\mathbf{4 5} \mathbf{~ m i n}$. To distinguish internalized bacteria from those bound to the surface, an assay was developed in which sodium azide was used to release surface-bound particles. Surprisingly, surface particle release appears to be independent of myosin II function. Using this assay it was shown that the uptake of bacteria into cells is extremely rapid. After 1 min incubation, $\mathbf{2 0} \%$ of the signal is derived from internalized bacteria. The proportion of the signal from internalized bacteria increases gradually and reaches $\mathbf{5 0} \%$ at steady state. This assay will be useful in investigations of the molecular machinery of phagocytosis and post-internalization vesicle trafficking.

Keywords: phagocytosis, myosin II

\section{INTRODUCTION}

Phagocytosis in eukaryotic organisms is essential for the clearance of invading bacteria, cell debris and apoptotic cells (Franc et al., 1999; Greenberg, 2001). Many cell types are able to phagocytose particles; however, some cells, like neutrophils and monocytes of the immune system, are considered professional phagocytes because of their robust and versatile phagocytic capabilities. Receptors on the surface of phagocytic cells bind particles and initiate a signal transduction cascade which leads to actin polymerization. Particles are then internalized into phagosomes and these later fuse with lysosomes to initiate degradation of their contents. Some of the molecular details of these processes have been

Abbreviations: DIC, differential interference contrast; DsRed-Ec, DsRed Escherichia coli; FIU, fluorescence intensity unit(s). revealed, but many aspects are still unclear (Aderem \& Underhill, 1999; Kwiatkowska \& Sobota, 1999).

Phagocytic ability is not limited to multicellular eukaryotes. Simpler organisms, like the social amoeba Dictyostelium, are very agile phagocytes. The process of particle uptake in Dictyostelium looks remarkably similar to macrophage phagocytosis (Aizawa et al., 1997; Rupper \& Cardelli, 2001). The evidence suggests that phagocytosis is driven by a receptor-activated signal transduction cascade leading to the actin cytoskeleton, similar to the system observed in mammalian cells. However, in Dictyostelium it is possible to take a genetic approach to understanding the process of phagocytosis (Cornillion et al., 2000; Janssen \& Schleicher, 2001; Robinson \& Spudich, 2000). To initiate a genetic analysis of phagocytosis, it was important to have a robust and versatile method of characterizing the process. In this paper we report the use of Escherichia coli expressing the $28 \mathrm{kDa}$ fluorescent protein DsRed 
(Baird et al., 2000) for a Dictyostelium phagocytosis assay. This phagocytosis substrate provides a bright signal that is digestible by cells, resistant to photobleaching and provides a fluorescence emission that is stable at physiological pH (Aubry et al., 1993; Baird et al., 2000). To separate the process of binding from internalization, we have added a washing step that removes bound bacteria from the surface of the cells. This optimized assay allows examination of rates of surface binding, internalization and degradation of prey particles

\section{METHODS}

Cell culture. The Dictyostelium discoideum cell lines used in this study include wild-type parental cell lines AX2 (Watts \& Ashworth, 1979), AX4 (Knecht et al., 1986), AX3K (Loomis, 1971) and NC4-A2 (Shelden \& Knecht, 1995); the mutant cell lines HS1 (Ruppel et al., 1994), lacking myosin II heavy chain $\left(m b c A^{-}\right)$, and HTD17-1, lacking myosin VII and its nonhomologous recombinant control G1-21 (Titus, 1999). All strains were grown in $100 \mathrm{~mm}$ plastic Petri dishes with $10 \mathrm{ml}$ HL-5 medium (Shelden \& Knecht, 1995). Two days before an assay, cells were inoculated at $1 \times 10^{5}$ cells ml $^{-1}$ to harvest cells at approximately $1 \times 10^{6}$ cells $\mathrm{ml}^{-1}$ for the assay.

DsRed-expressing E. coli. Fluorescent bacteria for phagocytosis assays were prepared by introducing a DsRed expression vector into E. coli cells. The E. coli line XL-1 Blue MFR' (Stratagene) was transformed with the vector pDG75 (kindly provided by Dr Daniel Gage, University of Connecticut, Storrs, CT, USA). The vector was created by amplifying the DsRed gene from pDsRed (Clontech) using a primer containing a ribosome-binding site (as described in Bringhurst et al., 2001) and the fragment was cloned into pGemTeasy (Promega). E. coli cells containing the vector were grown in LB medium with $1.0 \mathrm{mM}$ IPTG and $75 \mathrm{mg}$ ampicillin $\mathrm{ml}^{-1}$ at $37^{\circ} \mathrm{C}$ with shaking. After the cells reached stationary phase the cultures were shaken for an additional $24 \mathrm{~h}$ during which time the DsRed became maximally fluorescent. Cultures were concentrated by centrifugation and resuspended at an $\mathrm{OD}_{600}$ of 8.0 in $20 \mathrm{mM}$ sodium phosphate buffer $(\mathrm{pH} \mathrm{6.3)}$ and stored at $4{ }^{\circ} \mathrm{C}$ for no more than 3 weeks $(1: 200$ dilution in above buffer measured in a BioRad SmartSpec 3000).

Phagocytosis assay. Dictyostelium cells were harvested by centrifugation in the cold for $5 \mathrm{~min}$ at $200 \mathrm{~g}$, washed once in $20 \mathrm{mM}$ sodium phosphate buffer, $\mathrm{pH} 6 \cdot 3$, and resuspended at a concentration of $4 \times 10^{6}$ cells ml $^{-1}$. Cells were then incubated in $20 \mathrm{mM}$ sodium phosphate buffer, $\mathrm{pH} 6 \cdot 3$, at $22^{\circ} \mathrm{C}$ and shaken at 130 r.p.m. in a $50 \mathrm{ml}$ flask for $15 \mathrm{~min}$. After this initial incubation, $2 \times 10^{10}$ DsRed E. coli (DsRed-Ec) cells were added for every $4 \times 10^{6}$ Dictyostelium cells. Alternatively, $2 \times 10^{10}$ YG fluorescent plain latex beads (Polysciences) were used. DsRed-Ec or latex beads were briefly sonicated and vortexed vigorously to break-up any aggregates before addition to the Dictyostelium suspension. This gave a final ratio of 5000 prey particles to each Dictyostelium cell. At each time point in the assay, $0.5 \mathrm{ml}$ cells were transferred from the flask to $5 \mathrm{ml}$ ice cold $20 \mathrm{mM}$ sodium phosphate buffer in a $15 \mathrm{ml}$ conical tube. Cells were washed free of unbound bacteria by centrifugation at $200 \mathrm{~g}$ for $5 \mathrm{~min}$ at $4{ }^{\circ} \mathrm{C}$ in a swinging bucket rotor (Beckman JH6). The pellet was then resuspended in $5 \mathrm{ml}$ fresh buffer by vortexing for $5 \mathrm{~s}$ at maximum speed. The pellets were washed two additional times and after the third wash the pellet was resuspended in $200 \mu \mathrm{l}$ buffer and transferred to a 96-well microtitre plate (Falcon). Fluorescence was assessed in a plate fluorimeter (CytoFluor Series 400; PerSeptive Biosystems) using $530 \mathrm{~nm}$ excitation and 580 emission for the DsRed signal or $485 \mathrm{~nm}$ excitation, $508 \mathrm{~nm}$ emission for YG beads. Each time point was done in triplicate from the same flask unless otherwise indicated. Background signal was established by adding $2 \times 10^{10}$ DsRed-expressing bacteria or $2 \times 10^{10}$ YG beads per millilitre of $20 \mathrm{mM}$ sodium phosphate buffer to flasks without cells and prepared as above.

To remove surface-bound bacteria or beads, the first two washes were performed in $20 \mathrm{mM}$ sodium phosphate buffer containing $5 \mathrm{mM}$ sodium azide, a modification of the method of Glynn (1981). This azide concentration causes release of surface-bound bacteria and beads, but does not significantly reduce cell viability if it is subsequently washed out (data not shown). The final wash did not contain azide and the cells were resuspended in buffer without sodium azide before they were analysed in the fluorescent plate reader.

Binding without uptake. To measure binding in the absence of uptake, flasks containing Dictyostelium were incubated in an ice bucket of packed shaved ice on a rotary shaker $(100$ r.p.m.) for $15 \mathrm{~min}$ before ice-cold bacteria were added. The mixture was then incubated on ice while shaking for $10 \mathrm{~min}$ and then washed as described above. All steps were performed at as close to $0^{\circ} \mathrm{C}$ as possible.

DsRed breakdown. To measure breakdown of fluorescent bacteria, cells were allowed to take up bacteria for $30 \mathrm{~min}$ as described above. The cells were then washed twice in ice-cold sodium phosphate buffer, $\mathrm{pH} 6 \cdot 3$, with $5 \mathrm{mM}$ sodium azide and once in buffer without sodium azide. Washed cells were

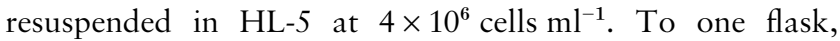
unlabelled Klebsiella aerogenes was added to a final concentration of $1 \times 10^{10} \mathrm{ml}^{-1}$ and an equal volume of buffer was added to the other. The cells were shaken at 130 r.p.m. and aliquots were removed at specified time points and centrifuged at $2400 \mathrm{~g}$ for $5 \mathrm{~min}$ at $4{ }^{\circ} \mathrm{C}$ to separate cells from any secreted DsRed-labelled material. Three hundred microlitres of supernatant was removed and added to a 96-well microtitre plate. The rest of the supernatant was discarded and then the cells were resuspended and added to another well. The fluorescence of the cells and supernatant was measured on a fluorescence plate reader as described above.

Confocal microscopy. Images of cells and bound bacteria were acquired using a Bio-Rad MRC600 laser scanning confocal microscope. To minimize internalization of bound particles during imaging, the cells were placed in $20 \mathrm{mM}$ sodium phosphate buffer in a Rose chamber cooled by a 20/20 Technologies stage controller set at $0{ }^{\circ} \mathrm{C}$ on the stage of a Zeiss Axiovert Microscope. Dual channel imaging was used with a rhodamine filter to visualize the DsRed bacteria and differential interference contrast (DIC) to image the whole cells and bound particles. Optical sections of the rhodamine channel were made using a $\times 631.25 \mathrm{NA}$ oil immersion objective. Z-Series images were collected at $0.5 \mu \mathrm{m}$ intervals. Single slices from the Z-stacks are presented with the two channels overlaid. The DsRed bacteria are pseudocoloured red in the merged image. Images were processed with $\mathrm{NIH}$ image (developed at the US National Institutes of Health and available at http://rsb.info.nih.gov/nih-image/) and Photoshop (Adobe).

\section{RESULTS}

\section{Uptake of DsRed-Ec by Dictyostelium}

Most phagocytosis assays utilize either dead, chemically labelled bacteria or yeast, or fluorescent beads as a 


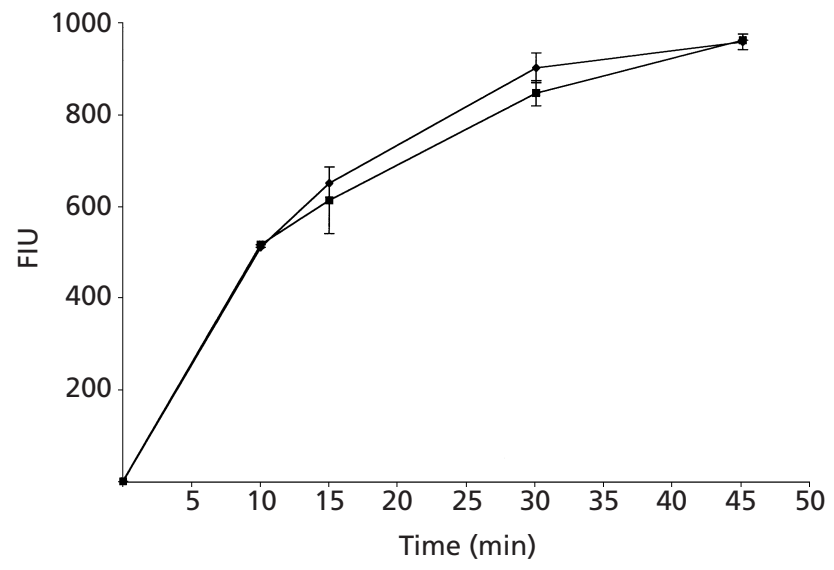

Fig. 1. Comparison of fluorescent latex beads and DsRed-Ec as phagocytosis uptake markers. After the indicated incubation time the suspensions of DsRed-Ec or fluorescent latex beads and AX2 cells were washed twice and the fluorescence measured in a plate fluorimeter. Each time point is a mean of two independent samples from the same bacteria/AX2-beads mixture. Maximum fluorescence intensity unit (FIU) values were normalized to 1000. $\bullet$, AX2-DsRed-Ec; $\mathbf{\square}$, AX2-beads.

target particle for phagocytosis. We have developed an assay using live bacteria expressing the fluorescent protein DsRed (DsRed-Ec), allowing us to assess both uptake and processing of bacteria by cells. When cells were shaken in suspension with DsRed-Ec, cells rapidly began to take up bacteria and cell-associated fluorescence continued to increase until a plateau was reached after about $30 \mathrm{~min}$ (Fig. 1). If fluorescent latex beads were used instead of bacteria, the kinetics of the process were similar (Fig. 1).

There are numerous 'wild-type' Dictyostelium cell lines used for making mutants that are subsequently assessed for defects in phagocytosis. Therefore, it was of interest to use this assay to determine if all of these cell lines were equally proficient at phagocytosis. The results indicate that there are differences in the rate of phagocytosis between these commonly used strains. The most striking difference is that AX4 is less efficient at internalizing DsRed-Ec than NC4-A2, AX2 or AX3-K (Fig. 2a). The AX4 cell line is also less efficient at internalizing latex beads than the other wild-type cell lines (Fig. 2b). The decision to use AX2 for subsequent studies was based on its robust ability to phagocytose both DsRed-Ec and beads.

\section{Contribution of surface-bound bacteria to total signal}

The process of phagocytosis can be divided into two phases: binding and internalization. Other phagocytosis assays have used trypan blue to quench the fluorescent signal from bacteria still on the surface to distinguish this signal from internalized bacteria (Maniak et al., 1995). Since the DsRed-Ec cells are alive, quenching will not work, so we sought an alternative method. Since
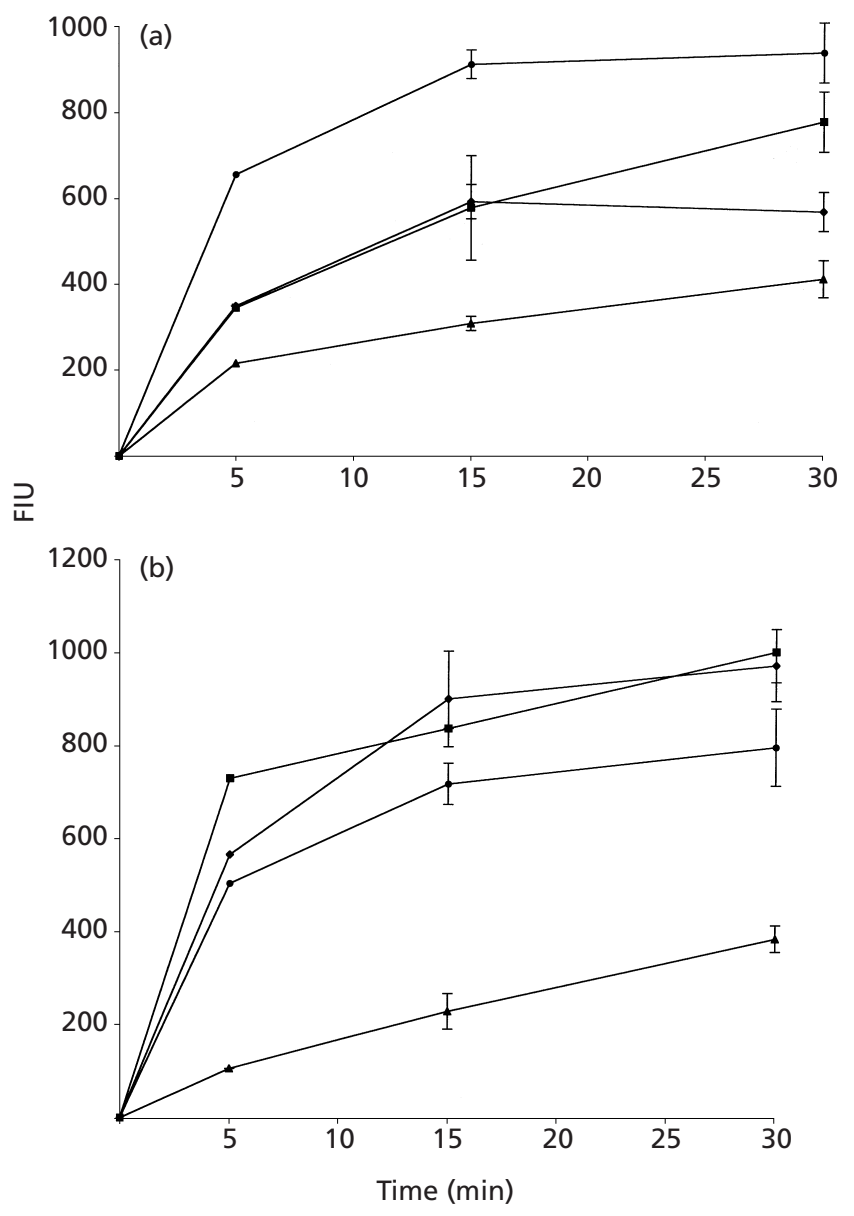

Fig. 2. Comparisons of standard axenic 'wild-type' cell lines in the phagocytosis assay. After the indicated incubation time suspensions of DsRed-Ec (a) or fluorescent latex beads (b) and cells were washed twice and the fluorescence measured in a plate fluorimeter. Each time point is a mean of two independent samples from the same mixture. Maximum FIU values were normalized to $1000 . \diamond, A X 2 ; \mathbf{\square}, A X 3 K ; \boldsymbol{\Delta}, A X 4 ; \bullet$, NC4-A2.

cells undergo a cortical contraction and round up when treated with sodium azide (Pasternak et al., 1989), it seemed possible that this process might release bacteria bound to the surface without affecting bacteria already internalized into phagosomes. Cells can recover completely from azide treatment, so it appears not to damage them irreversibly.

Several experiments were performed to confirm that azide was removing surface-bound bacteria. To visualize the effects of azide treatment, cells were incubated for various times with DsRed-Ec and then washed with or without azide. The cells were then imaged with a confocal microscope. This allowed us to use optical sectioning to determine if bacteria were near the surface of the cell or inside. Cells incubated for $5 \mathrm{~min}$ or more had many bacteria associated with the surface when washed with buffer alone and some bacteria appeared to 

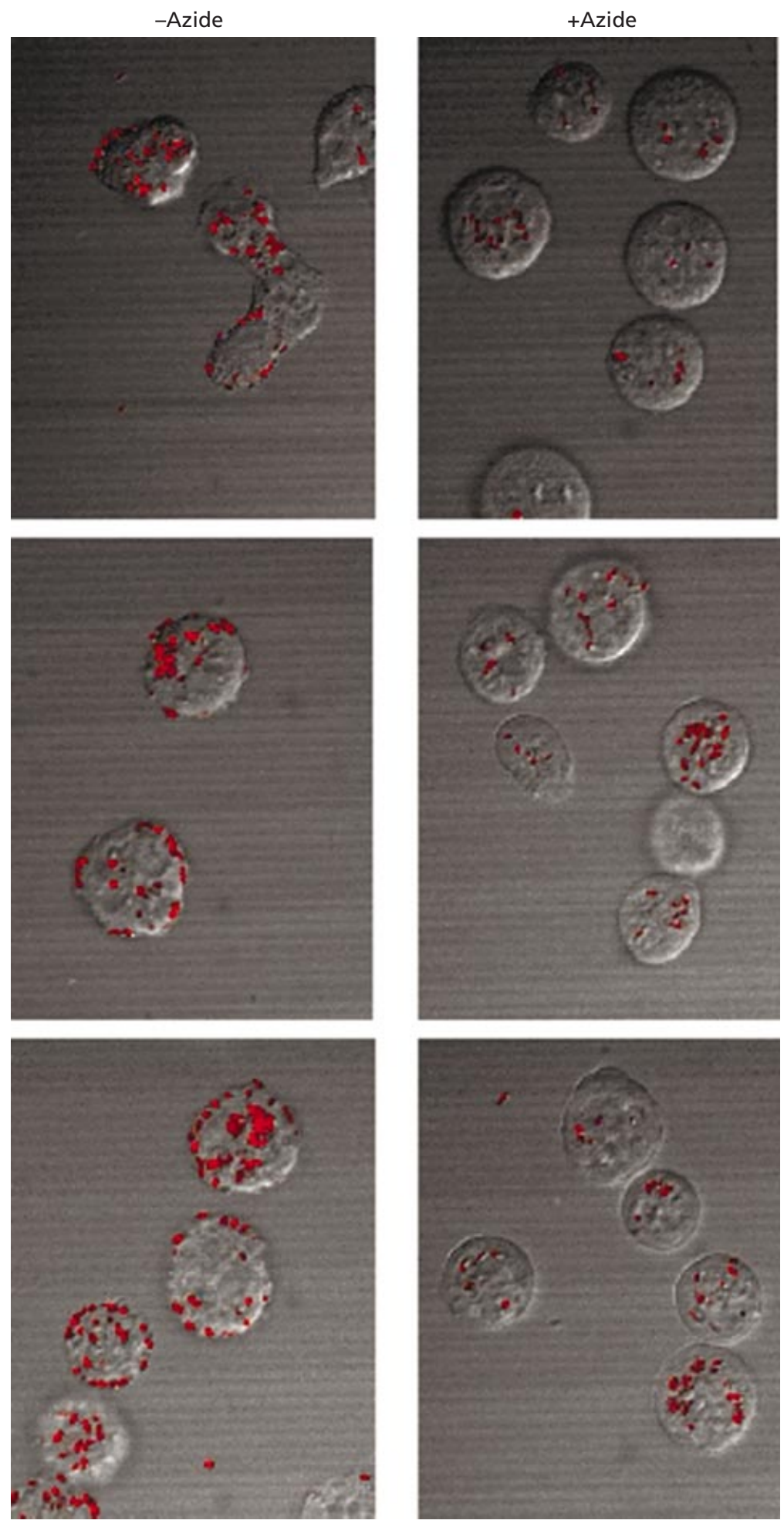

$5 \min$

$10 \mathrm{~min}$

$40 \mathrm{~min}$
Fig. 3. Azide washing removes DsRed-Ec from the surface of cells. After the indicated incubation time the suspension of DsRed-Ec and AX2 cells was washed twice with the indicated treatment and once in plain sodium phosphate buffer. Images were acquired by Z-sectioning through the cells using a confocal microscope. Single representative focal planes near the centre of the cells are shown which make it easy to distinguish the cell perimeter. The DIC image of the cells was merged with the rhodamine (DsRed) channel in each section and the DsRed-Ec are pseudocoloured red. have been internalized (Fig. 3). When washed with azide, all the DsRed-Ec cells were found inside the cell. When cells that had taken up bacteria for $40 \mathrm{~min}$ were examined, there was a significant increase in the amount of bacteria associated with the surface as well as inside the cell. All of the surface-bound bacteria appeared to have been removed by the azide wash.
When two azide washes were employed in the assay, we observed a reduction in the fluorescence signal consistent with our visual observation. After $1 \mathrm{~min}$ of uptake, when most of the bacteria are expected to be surfaceassociated, azide treatment removed more than $80 \%$ of the fluorescence signal (Fig. 4b). The proportion of the signal washed off decreased over time until it reached a 

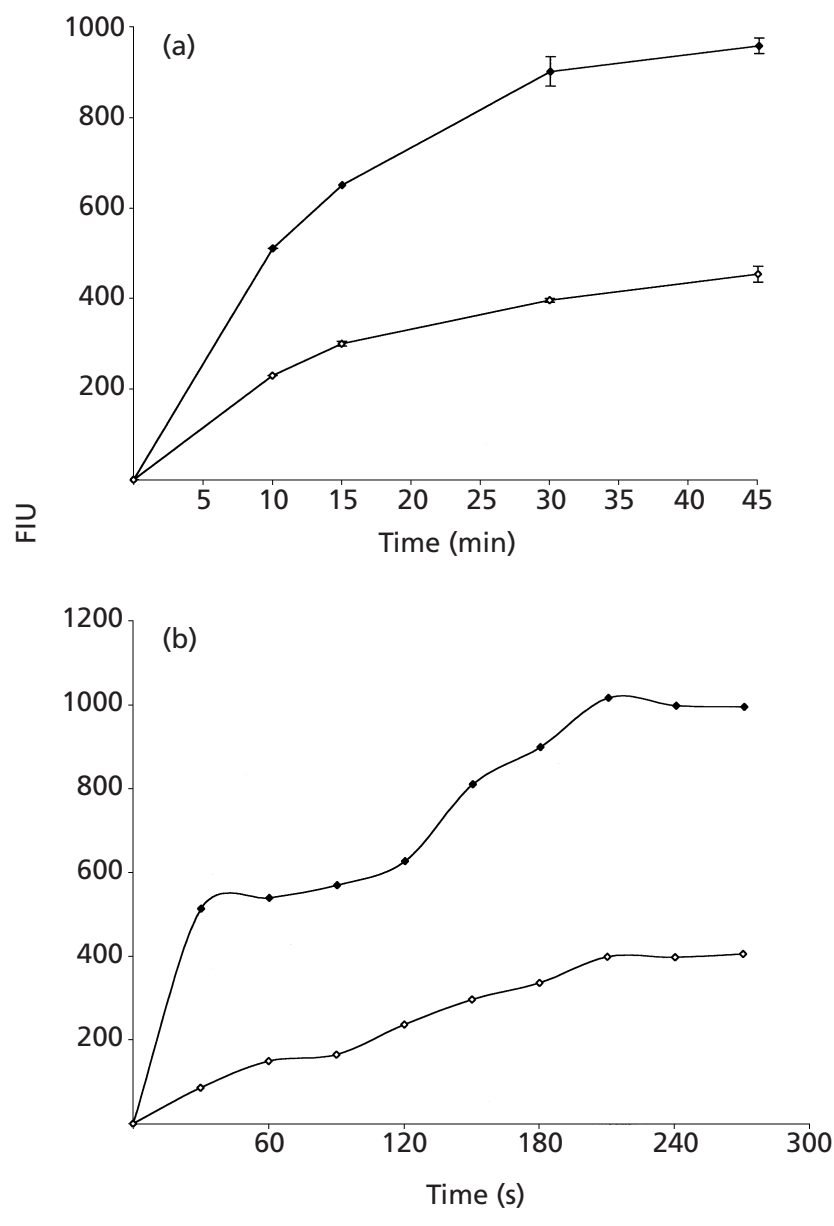

Fig. 4. Surface-bound DsRed-Ec accounts for $50 \%$ of total fluorescence at equilibrium. (a) After the indicated incubation time, the suspension of DsRed-Ec and AX2 cells was washed twice with either sodium phosphate buffer $(\diamond)$ or buffer containing azide $(\diamond)$ and once in sodium phosphate buffer. Each time point is a mean of three independent samples from the same bacteria/AX2 mixture. (b) Duplicate samples taken during the first $300 \mathrm{~s}$ of uptake. At the $30 \mathrm{~s}$ time point, the surface-bound bacteria account for approximately $80 \%$ of the signal. After $40 \mathrm{~min}$ incubation the azide-releasable signal is $50 \%$ of the total signal. Maximum FIU valves were normalized to 1000 . $\diamond$, Total bacteria; $\diamond$, internalized bacteria.

plateau at approximately $50 \%$ of the total signal (Fig. 4a). This number represents the proportion of the signal that is due to surface-bound bacteria. Similar results were obtained when fluorescent latex beads were used as substrate (data not shown).

To confirm that the azide wash was removing surfacebound bacteria, the assay was performed under conditions where all of the bacteria should be surfaceassociated. Cells were allowed to bind DsRed-Ec at $0{ }^{\circ} \mathrm{C}$, which prevents internalization (Rabinovitch, 1967). When these cells were washed in $5 \mathrm{mM}$ azide, $90 \%$ of the fluorescence was lost. By varying the wash conditions, the requirements for azide-induced release were determined. At azide concentrations below $0.05 \mathrm{mM}$, the release was incomplete (data not shown).

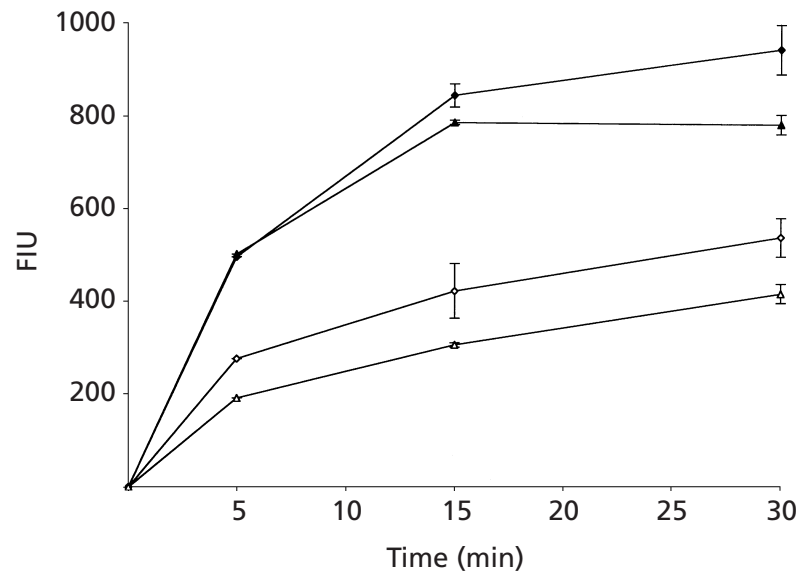

Fig. 5. Release of bacteria from the surface of Dictyostelium is not dependent on myosin II. After the indicated incubation times the suspension of DsRed-Ec and AX2 or $m h c A^{-}$cells was washed twice with the indicated treatment and once in plain sodium phosphate buffer. Each time point is a mean of three independent samples from the same cell mixture. Maximum FIU valves were normalized to $1000 . \diamond, A X 2 ; \diamond, A X 2$ with azide; $\triangle, m h c A^{-}$cells; $\boldsymbol{\Delta}, m h c A^{-}$cells with azide.

An azide concentration of $5 \mathrm{mM}$ gave consistent results and did not compromise cell viability. It is important to note that the azide-mediated release required some mechanical agitation to induce the release of particles. If azide was added to cells while they were being imaged on the microscope stage, no release was observed. Likewise, gentle agitation when resuspending pellets in the standard assay did not remove surface-bound bacteria. Only when cells were vigorously vortexed was release observed. These data indicate that azide treatment reduces the tightness of binding of bacteria to the surface, but does not in and of itself cause bacterial release. Dictyostelium washed with azide after uptake of rhodamine dextran, as a fluid phase marker, showed no reduction in fluorescent signal when compared with untreated cells (data not shown). This result suggests that azide is not stimulating release of intracellular vesicles.

The rounding and detachment of cells from surfaces in the presence of azide are mediated by myosin II, since cells lacking myosin II contractile activity remain attached to the substrate when exposed to azide (Pasternak et al., 1989). To assess if the release of bound bacteria from the surface of the cell was mediated by a generalized cell shape change, we analysed the effect of azide on phagocytosis by mutant cells lacking myosin II heavy chain $\left(m b c A^{-}\right) . m b c A^{-}$cells were able to phagocytose bacteria nearly as well as wild-type cells (Fig. 5). Surprisingly, the release of bacteria from $m b c A^{-}$cells was equivalent to that of wild-type cells (Fig. 5). This result indicates that myosin II is not required for phagocytosis of bacteria and that the azide-induced bacterial release is not functioning through a generalized cell shape change or other myosin II-dependent mechanism. 


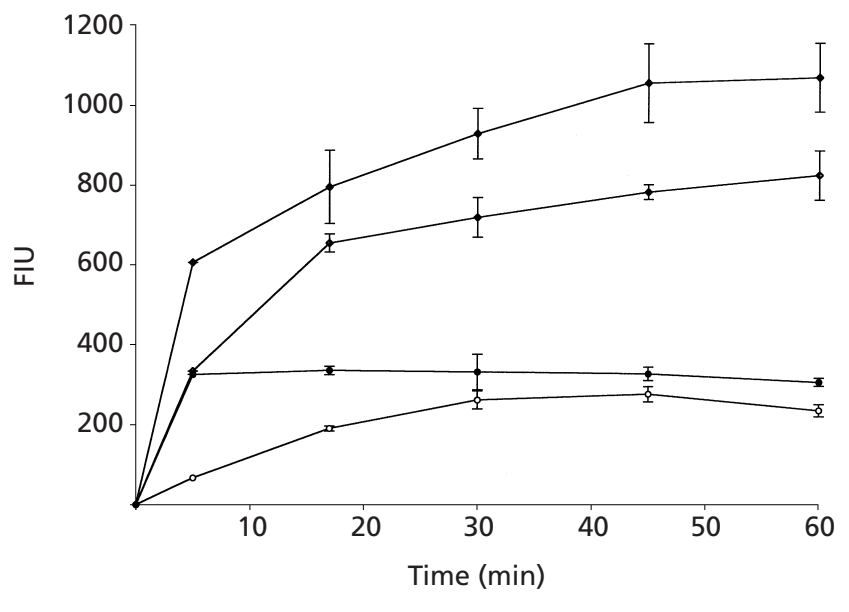

Fig. 6. Release of bacteria from the surface of Dictyostelium is not dependent on myosin VII. After the indicated incubation times the suspension of DsRed-Ec and myosin VII null cells or control cells was washed twice with the indicated treatment and once in plain sodium phosphate buffer. Each time point is a mean of three independent samples from the same cell mixture. Myosin VII null cells; $\bigcirc$, myosin VII null cells with azide; $\diamond$, control cells; $\diamond$, control cells with azide.

It is essential that this modified assay should be able to identify cells defective in phagocytosis. To verify that this assay is able to identify cells defective in phagocytosis and further explore the sodium-azide-mediated release, phagocytosis by myosin VII null cells (DdMVII null) was examined. These cells are known to exhibit a substantially reduced rate of phagocytosis of both latex beads and fluorescently labelled yeast (Titus, 1999; Tuxworth et al., 2001). The role of myosin VII is to mediate adhesion during the early stages of phagocytosis (Tuxworth et al., 2001). This makes DdMVII null cells interesting candidates for testing the DsRed-Ec assay. DdMVII nulls cells have a substantially slower rate of uptake of DsRed-Ec when compared with control cells (Fig. 6). This finding is consistent with published results (Titus, 1999; Tuxworth et al., 2001) and verifies the ability of this assay to identify cells defective in phagocytosis. When the DdMVII null cells were washed with sodium azide, the amount of signal was reduced (Fig. 6). This suggests that the binding of particles to DdMVII cells is sodium-azide-sensitive and that myosin VII is not responsible for the azide-induced release of surface-bound particles.

\section{Degradation of DsRed-Ec}

One of the advantages of using DsRed-Ec instead of dyelabelled bacteria is that the fluorescent protein should be eventually degraded by the phagolysosomal system. Therefore, it is possible to directly assess the processing of internalized material. To measure the degradation rate of DsRed-Ec, a pulse-chase experiment was performed. Dictyostelium cells were allowed to internalize DsRed-Ec for $30 \mathrm{~min}$ and then the free and surfacebound bacteria were removed; the incubation was

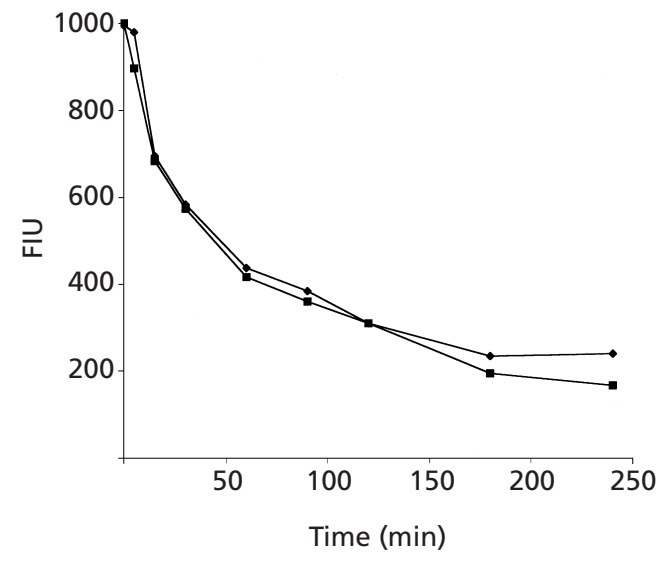

Fig. 7. Degradation of DsRed-fluorescent signal by $A X 2$. AX2 cells were allowed to phagocytose DsRed-Ec for $30 \mathrm{~min}$ in HL-5 medium. Cells were then washed twice with $5 \mathrm{mM}$ sodium azide to remove surface-bound bacteria and then once with buffer without sodium azide. To prevent starvation conditions from initiating development, the cells were incubated with either unlabelled $K$. aerogenes $(\diamond)$ or HL-5 medium ( $\square$ ) during the chase. The fluorescence of the cell pellet was measured at various times after initiating the chase. Initial FIU values were normalized to 1000 .

continued with either unlabelled bacteria or HL5 growth medium. The DsRed fluorescent signal associated with the cells decreased continuously during the chase with a half-life of $45 \mathrm{~min}$ (Fig. 7). The presence of bacteria in the chase condition does not appear to alter the rate of DsRed-Ec degradation. No signal was detected in the chase medium, indicating that the DsRed protein is degraded prior to release of any material from the cells (data not shown).

\section{DISCUSSION}

Phagocytosis and digestion of prey particles is a multistep process. It involves binding of the particle by a surface receptor, changes in the actin cytoskeleton that lead to the particle becoming surrounded by membrane and then membrane fusion and internalization of the particle into phagosomal vesicles (Greenberg, 1995). The phagosome is then processed by the lysosomal pathway, leading to degradation of the vesicle contents. To investigate the kinetics of phagocytosis it is necessary to distinguish surface-bound particles from internalized particles. The use of a sodium azide wash allows us to make this distinction. Our results show that a surprisingly large proportion of the signal $(50 \%)$ in the standard phagocytosis assay is derived from surfacebound bacteria. If not taken into account, this surfacebound fraction leads to an overestimation of the amount of phagocytosis and may mask some of the kinetics of internalization.

The mechanism of sodium-azide-mediated release of surface-bound particles remains unclear. It may act through depletion of ATP from the cells; however, the fact that particles are released even when the treatment 
is done in the cold argues that the phenomenon may be more complex. In addition, the release of surface-bound particles requires some mechanical agitation. Since both beads and bacteria are released by azide and the uptake of these particles is mediated by different receptors, it seems unlikely that the azide release is due to a direct effect on receptor affinity. A more likely explanation is that azide has an effect on cytoskeletal protein(s) that modulate receptor function. It is clear that the release of particles from the surface of the cells is not mediated by myosin II, although myosin II has been found in the phagocytic cup of lung macrophages (Stendahl et al., 1980). Cells lacking myosin II function show the same azide-induced release of bacteria as wild-type cells. Myosin VII is present at the early stages of phagocytic cup formation and is another possible candidate for the molecule affected by sodium azide (Tuxworth et al., 2001). Mutants that lack myosin VII are defective in uptake of DsRed-Ec, but the small amount of surface binding that does occur is partially azide-sensitive, suggesting that myosin VII is not essential for azide release. When cells are treated with 2,4-dinitrophenol, an uncoupler of oxidative phosphorylation, the cells round up and the cortical actin filament system is disrupted (Jungbluth et al., 1994). The rearrangement of the actin cytoskeleton induced by starving cytoskeletal components for ATP combined with the shear forces generated by vortexing the cells may cause the release of incompletely internalized bacteria.

There are several advantages to using E. coli expressing DsRed as a phagocytosis uptake marker. A major consideration is alteration of the surface chemistry of the bacteria. The surface chemistry of the prey particle is important to how Dictyostelium interacts with the particle (Cohen et al., 1994; Cornillion et al., 2000; Vogel et al., 1980). Since the DsRed bacteria are collected from growth medium and used for the assay, changes in surface chemistry caused by handling are negligible. The use of live E. coli also prevents loss of any attractant factors or signals that are secreted by living bacteria and any modification to the surface of the cell wall that is the result of preparation and labelling of bacteria using a heat killing step. A second advantage is that the fluorescence emission from DsRed protein is very $\mathrm{pH}$ stable (Baird et al., 2000). The stability of DsRed works in tandem with live E. coli which have an intact membrane, so the DsRed is protected from the environment of the phagolysosome until the bacterial membrane is disrupted. This situation differs from artificially labelled bacteria, where the fluorescence marker is accessible to the acidic environment of the phagosome immediately after internalization. Loss of the E. coli-derived DsRed fluorescence signal is likely to be the direct result of the degradation of the DsRed protein and not a $\mathrm{pH}$ quenching effect. A final advantage is that unlike chemical labelling procedures, it is very simple and inexpensive to prepare large amounts of bacteria for phagocytosis assays. Thus E. coil expressing DsRed has great utility as a target particle for phagocytosis assays. Expression of the DsRed protein in other bacteria with known surface characteristics will give us the opportunity to examine the role of surface chemistry on phagocytosis.

Using DsRed-Ec, we observed a half-life of the fluorescence signal of $45 \mathrm{~min}$. This suggests that after $30 \mathrm{~min}$ incubation, when the fluorescence signal begins to plateau, we are observing the equilibrium between uptake and degradation of the DsRed signal. In this plateau period, the difference between the sodium-azidewashed samples and untreated samples indicates the binding capacity of the surface of the cell for bacteria. This binding capacity represents approximately $50 \%$ of the total signal at equilibrium. This result suggests that binding of bacteria is not the rate-limiting step in phagocytosis, since abundant surface-bound bacteria are available for internalization. The ability to remove bacteria from the surface with azide is critical to the efforts we have initiated to genetically screen for phagocytosis mutants. Mutants that bind, but do not internalize bacteria would be difficult to identify if we were not able to remove the surface-bound bacteria.

The combination of DsRed with a mild sodium azide wash to release surface-bound bacteria produces a versatile phagocytosis assay. This assay is well suited for examination of the kinetics of particle binding and uptake, and for screening for mutants defective in both the binding and subsequent stages of phagocytosis. Understanding the biochemical mechanisms of phagocytosis will be aided by our ability to separate the process into discrete steps and to identify the molecular components involved.

\section{ACKNOWLEDGEMENTS}

The authors would like to acknowledge the generous gift of the pDG75-expressing E. coli strain by Dr Daniel Gage, and the generous gift of HTD17-1 and helpful comments of Drs Margaret Titus and Richard Tuxworth. We wish to thank Dr Charles Giardina for the use of his plate fluorimeter and Catherine Green for assistance with cell culture and general lab support. This work was supported by a grant from the NIH (GM-40599) and a University of Connecticut Research Foundation Award.

\section{REFERENCES}

Aderem, A. \& Underhill, D. M. (1999). Mechanism of phagocytosis in macrophages. Annu Rev Immunol 17, 593-623.

Aizawa, H., Fukui, Y. \& Yahara, I. (1997). Live dynamics of Dictyostelium cofilin suggests a role in remodeling actin latticework into bundles. J Cell Sci 110, 2333-2344.

Aubry, L., Klein, G., Martiel, J. L. \& Satre, M. (1993). Kinetics of endosomal $\mathrm{pH}$ evolution in Dictyostelium discoideum amoebae. J Cell Sci 105, 861-866.

Baird, G. S., Zacharias, D. A. \& Tsien, R. Y. (2000). Biochemistry, mutagenesis, and oligomerization of DsRed, a red fluorescent protein from coral. Proc Natl Acad Sci US A 97, 11984-11989.

Bringhurst, R. M., Cardon, Z. G. \& Gage, D. J. (2001). Galactosides in the rhizosphere: Utilization by Sinorhizobium meliloti and development of a biosensor. Proc Natl Acad Sci USA 98, $4540-4545$. 
Cohen, J. C., Bacon, R., Clarke, M., Joiner, K. \& Mellman, I. (1994). Dictyostelium discoideum mutants with conditional defects in phagocytosis. J Cell Biol 126, 955-966.

Cornillion, S., Pech, E., Benghezal, M., Ravanel, K., Gaynor, E., Letourneur, F., Brucker, F. \& Cosson, P. (2000). Phg1p is a ninetransmembrane protein superfamily member involved in Dictyostelium adhesion and phagocytosis. J Biol Chem 275, 34287-34292.

Franc, N. C., White, K. \& Ezekowitz, R. A. (1999). Phagocytosis and development: back to the future. Curr Opin Immunol 11, 47-52.

Glynn, P. J. (1981). A quantitative study of the phagocytosis of Escherichia coli by myxamoebae of the slime mould Dictyostelium discoideum. Cytobios 30, 153-166.

Greenberg, S. (1995). Signal transduction of phagocytosis. Trends Cell Biol 5, 93-99.

Greenberg, S. (2001). Diversity in phagocytic signalling. J Cell Sci 114, 1039-1040.

Janssen, K. P. \& Schleicher, M. (2001). Dictyostelium discoideum: A genetic model system for the study of professional phagocytes Profilin, phosphoinositides and the $1 \mathrm{mp}$ gene family in Dictyostelium. Biochim Biophys Acta 1525, 228-233.

Jungbluth, A., von Arnim, V., Biegelmenn, E., Humbel, B., Schweiger, A. \& Gerisch, G. (1994). Strong increase in the tyrosine phosphorylation of actin upon inhibition of oxidative phosphorylation: correlation with reversible rearrangements in the actin skeleton of Dictyostelium cells. J Cell Sci 107, 117-125.

Knecht, D. A., Cohen, S. M., Loomis, W. F. \& Lodish, H. F. (1986). Developmental regulation of Dictyostelium discoideum actin gene fusions carried on low-copy and high-copy transformation vectors. Mol Cell Biol 6, 3973-3983.

Kwiatkowska, K. \& Sobota, A. (1999). Signaling pathways in phagocytosis. Bio Essays 21, 422-431.

Loomis, W. F. (1971). Sensitivity of Dictyostelium discoideum to nucleic acid analogues. Exp Cell Res 64, 484-486.

Maniak, M., Rauchenberger, R., Albrecht, R., Murphy, J. \& Gerisch, G. (1995). Coronin involved in phagocytosis: Dynamics of particle-induced relocalization visualized by a green fluorescent protein Tag. Cell 83, 915-924.
Pasternak, C., Spudich, J. A. \& Elson, E. L. (1989). Capping of surface receptors and concomitant cortical tension generated by conventional myosin. Nature 341, 549-551.

Rabinovitch, M. (1967). The dissociation of the attachment and ingestion phases of phagocytosis by macrophages. Exp Cell Res 46, 19-28.

Robinson, D. N. \& Spudich, J. A. (2000). Dynacortin, a genetic link between equatorial contractility and global shape control discovered by library complementation of a Dictyostelium discoideum cytokinesis mutant. J Cell Biol 150, 823-838.

Ruppel, K. M., Uyeda, T. Q. \& Spudich, J. A. (1994). Role of highly conserved lysine 130 of myosin motor domain. In vivo and in vitro characterization of site specifically mutated myosin. J Biol Chem 269, 18773-18780.

Rupper, A. \& Cardelli, J. (2001). Regulation of phagocytosis and endo-phagosomal trafficking pathways in Dictyostelium discoideum. Biochim Biophys Acta 1525, 205-216.

Shelden, E. \& Knecht, D. A. (1995). Mutants lacking myosin II cannot resist forces generated during multicellular morphogenesis. J Cell Sci 108, 1105-1115.

Stendahl, O. I., Hartwig, J. H., Brotschi, E. A. \& Stossel, T.P. (1980). Distribution of actin-binding protein and myosin in macrophages during spreading and phagocytosis. J Cell Biol 84, 215-224.

Titus, M. A. (1999). A class VII unconventional myosin is required for phagocytosis. Curr Biol 9, 1297-1303.

Tuxworth, R. I., Weber, X., Wessels, D. A., Addicks, G. C., Soll, D. R., Gerisch, G. \& Titus, M. A. (2001). A role for myosin VII in dynamic cell adhesion. Curr Biol 11, 318-329.

Vogel, G., Thilo, L., Schwarz, H. \& Steinhart, R. (1980). Mechanism of phagocytosis in Dictyostelium discoideum: Phagocytosis is mediated by different recognition sites as disclosed by mutants with altered phagocytic properties. J Cell Biol 86, 456-465.

Watts, D. J. \& Ashworth, J. M. (1979). Growth of myxamoebae of the cellular slime mould Dictyostelium discoideum in axenic culture. Biochem J 119, 171-174.

Received 10 July 2001; revised 8 October 2001; accepted 19 October 2001. 